\title{
Diseño exergoeconómico de la geometría de un calentador de aire tubular vertical de tres pasos
}

\section{Exergoeconomic Design of the Geometry of a Vertical, Three Steps, Tubular Air Heater}

\author{
J.J. Marín-Hernández \\ Facultad de Ingeniería Mecánica Eléctrica. \\ Universidad Veracruzana, Campus Xalapa. Veracruz, México. \\ E-mail:jmarin@uv.mx \\ M.L. González-PetitJean \\ Facultad de Ingeniería Mecánica Eléctrica. \\ Universidad Veracruzana, Campus Xalapa. Veracruz, México \\ E-mail:mgonzalezpetitjean@gmail.com \\ O.M. Cruz-Fonticiella \\ Facultad de Ingeniería Mecánica. \\ Universidad Central de las Villas. Cuba. \\ E-mail:ocf@uclv.edu.cu
}

(Recibido: noviembre de 2006; aceptado: septiembre de 2008)

\section{Resumen}

En este trabajo se aplica el método estructural termoeconómico para hallar el diseño geométrico óptimo de un calentador de aire tubular vertical, cuyo diseño original es ampliamente utilizado en calderas instaladas en ingenios azucareros. La función objetivo describe el costo total de operación asociado a las irreversibilidades debidas a la diferencia de temperaturas y la caída de presión en función de los principales parámetros geométricos. Dicha función objetivo es continua dentro del rango de análisis, aunque tiene un fuerte comportamiento no lineal, por lo tanto, para resolverla se utilizó el Toolbox de Optimización del Software MATLAB 6.5, a través de la función FMINCON, que utiliza un algoritmo de media escala, la Programación Secuencial Cuadrática (POS) y el método Cuasi-Newton. Se concluye, principalmente, con que el nuevo diseño propicia un decremento en los costos de operación del equipo de 1,97US\$/s al compararse con el de la caldera RETO45-28.

Descriptores: Termoeconomía, optimización geométrica, intercambiadores de calor.

\footnotetext{
Abstract

In this paper, the structural method of thermoeconomics is applied to find the optimal geometrical design to a vertical tubular air heater, whose original design is widely used on boilers installed on sugar mills. The objective function describes the running cost due to the temperature difference and pressure drop in function of the main geometrical parameters. The objective function is continuous in the interval of analysis and has a strong non linear behavior, then for solving it, the Optimization Toolbox from MATLAB 6.5 was used, through the function FMINCON, which uses an algorithm of medium
} 
scale, the Sequential Quadratic Programming (SQP) and the Cuasi Newton method. The new design reduces the running cost of equipment 1,97 US\$/s compared to the one of RETO $45-28$ system.

Keywords: Thermoeconomics, geometric optimization, heat exchangers.

\section{Introducción}

Uno de los retos más grandes que enfrenta la ciencia y la tecnología en los inicios del siglo XXI es diseñar sistemas energéticos más eficientes, tomando en cuenta consideraciones técnicas, económicas, normativas y medioambientales (Gong et al., 1997) que garanticen el suministro de bienes y servicios a la sociedad actual en base a los recursos limitados con los que cuenta el planeta, pero sin comprometer el consumo de las futuras generaciones (Cornelissen, 1997).

"Surge la termoeconomía como una técnica de análisis integradora que permite asignar costos, diagnosticar, mejorar y optimizar el diseño de sistemas térmicos tomando en cuenta no los valores subjetivos de las cosas sino el costo real, es decir, su costo exergético" (Valero, 2001).

Actualmente, dentro de la industria azucarera no se cuenta con los recursos suficientes para realizar grandes inversiones en la modernización de sus unidades, por ello, tienen prioridad aquellos proyectos que con un mínimo de recursos obtengan grandes beneficios en aprovechamiento de la energía. Los Equipos de Recuperación de Calor (ERC) de calderas adquieren un rol fundamental en este sentido.

Los Calentadores de Aire (CA) tubulares verticales de tres pasos en donde los gases circulan por el interior de los tubos y el aire realiza tres pasos en flujo cruzado a contracorriente, son equipos tradicionalmente utilizados como recuperadores de calor en calderas instaladas en ingenios azucareros; sin embargo, su diseño obedece sólo a criterios técnico-económicos basados en el primer principio de la termodinámica.

Varios autores (Holman, 1998; Hugot 1986; Incropera et al., 1996; entre otros) recomiendan amplios rangos de valores para definir su geometría (diámetros, pasos, espesores, velocidades, etc.), aunque se carece de la influencia del segundo principio.

Es por ello que en el presente trabajo se aplica el método estructural termoeconómico para hallar el diseño geométrico óptimo de un calentador de aire tubular vertical de tres pasos. Se concluye principalmente que la nueva propuesta provoca un decremento en los costos de operación del equipo de 1,97 US\$/s con respecto al diseño tradicional instalado en una caldera RETO 45-28.

\section{Desarrollo}

\section{Método}

Una vez conocidos los parámetros óptimos de frontera (flujo de gases, flujo de agua, flujo de aire, temperaturas de entrada y salida de las corrientes en análisis) del calentador de aire a partir de la primera etapa de optimización bajo una estrategia global (Marín, 2003), se determinan los parámetros geométricos óptimos a partir de una estrategia termoeconómica local de optimización, basada en los costos de producción asociados a las irreversibilidades totales.

La optimización geométrica de los calentadores de aire, idealmente debería llevarse a cabo en interacción termoeconómica con el resto de los elementos de la caldera, lo cual sería un procedimiento demasiado complejo.

Sin embargo, el carácter de la exergía como parámetro universal de la calidad de la energía para la asignación de costos, permite aproximadamente plantear una técnica de optimización local, siendo entonces la función objetivo definida por los costos totales de operación formados por los costos asociados a la irreversibilidad por transferencia de calor (IDT) y a los asociados a la caída de presión (ICP).

La ecuación 1 representa la función objetivo, la cual se desarrolla en función de parámetros de operación conocidos, constantes, propiedades termofísicas y de las variables geométricas libres de diseño.

El problema de optimización es:

$$
\operatorname{Min} \dot{C} I_{T}=c_{I}^{\Delta T} \dot{I}^{\Delta T}+c_{I}^{\Delta P} \dot{I}^{\Delta P}
$$

La ecuación 1 se puede representar desarrollada por 2 . 


$$
\begin{aligned}
& \dot{C} I_{T}=T_{0}\left\{c_{I}^{\Delta T}\left[C_{g a s} \ln \left(\frac{T_{\text {gas }, s}}{T_{\text {gas }, e}}\right)+C_{\text {air }} \ln \left(\frac{T_{\text {air }, s}}{T_{\text {air }, e}}\right)\right]\right. \\
& \left.+c_{I}^{\Delta P}\left[\dot{m}_{\text {gas }} R_{\text {gas }} \ln \left(\frac{P_{\text {gas }, e}}{P_{\text {gas }, s}}\right)+\dot{m}_{\text {air }} R_{\text {air }} \ln \left(\frac{P_{\text {air }, e}}{P_{\text {air }, s}}\right)\right]\right\}
\end{aligned}
$$

donde $C_{g a s}=\dot{m}_{g a s} c_{P, g a s}$

$\mathrm{y}_{\text {air }}=\dot{m}_{\text {air }} c_{P, \text { air }}$

Para este análisis se realizaron las siguientes consideraciones:

1. Las propiedades termo físicas son constantes y se determinan para el promedio de temperatura de las corrientes de entrada y salida.

2. El arreglo de tubos es al tresbolillo.

3. El número de Nusselt para el aire se calcula para una relación $\mathrm{S}_{T} / \mathrm{S}_{L}<2$.

4. Aunque se trata de un arreglo en flujo cruzado a contracorriente, la relación $\in=f(N T U, C, A r r)$ se aproximará a la de un arreglo a contracorriente.

5. El espesor de los tubos es invariable.

6. Los tubos son de acero al carbono.

7. El calentador de aire se encuentra bien aislado y no tiene pérdidas de calor al medio.

8. No existen infiltraciones en el calentador.

Las variables geométricas a optimizar son: el diámetro de los tubos, la longitud de los tubos, el número total de tubos y el número de tubos a lo ancho de la caldera. Para simplificar el problema, se consideró al paso transversal y longitudinal como dados. Las restricciones son expresadas por las ecuaciones 8 a 13. De forma indirecta se pueden determinar también los siguientes parámetros óptimos: la velocidad de gases y aire, el área de transferencia de calor, el coeficiente global de transferencia de calor, etc.

En el anexo se enumeran las ecuaciones necesarias que han sido utilizadas para hallar la IDT en función de los parámetros geométricos más importantes, expresada por 5 y 6 .

$$
\begin{gathered}
\dot{I}^{\Delta T}=T_{0}\left\{C_{g a s} \ln \left[1-C\left(\frac{1-e^{\pi D_{c} L N U\left(\frac{1}{C_{g a s}}-\frac{1}{C_{a i r}}\right)}}{1-C e^{\pi D_{c} L N U}\left(\frac{1}{C_{g a s}}-\frac{1}{C_{a i r}}\right)}\right)\left(1-\frac{T_{a i r, e}}{T_{g a s, e}}\right)\right]\right. \\
\left.+C_{\text {air }} \ln \left[1+C\left(\frac{1-e^{\pi D_{e} L N U\left(\frac{1}{C_{g a s}}-\frac{1}{C_{a i r}}\right)}}{1-C e^{\pi D_{c} L N U\left(\frac{1}{C_{g a s}}-\frac{1}{C_{\text {air }}}\right)}}\right)\left(\frac{T_{g a s, e}}{T_{\text {air }, e}}-1\right)\right]\right\}
\end{gathered}
$$

siendo:

$$
\begin{aligned}
& U=\left[4,51 \frac{\left(D_{e}-2 \varepsilon\right)^{1,8} \mu_{\text {gas }}^{0.5}}{c_{P, \text { aas }}^{0,3} k_{\text {gas }}^{0.7}}\left(\frac{N}{\dot{m}_{\text {gas }}}\right)^{0,8}\right. \\
& +0,23 \frac{\mu_{\text {air }}^{0,27}\left(D_{e}-2 \varepsilon\right)}{D_{e}^{0,6} c_{P, \text { air }}^{0,33} k_{\text {air }}^{0,66}}\left(\frac{\left(S_{T}-D_{e}\right)(L / 3) N_{A}}{\dot{m}_{\text {air }}}\right)^{0,6}\left(\frac{S_{T}}{S_{L}}\right)^{1 / 6} \\
& \left.+\frac{\left(D_{e}-2 \varepsilon\right) \ln \left(D_{e} /\left(D_{e}-2 \varepsilon\right)\right.}{2 k_{A C}}\right]^{-1}
\end{aligned}
$$

La ICP queda definida por 7, (ver anexo)

$$
\begin{aligned}
& \dot{I}^{\Delta P}=T_{0}\left\{\dot { m } _ { \text { gas } } R _ { \text { gas } } \operatorname { l n } \left[1+\frac{0.014 L}{\left(D_{e}-2 \varepsilon\right)^{5} \rho_{\text {gas }} P_{\text {gas }, 5}}\left(\frac{\dot{m}_{\text {gas }}}{N}\right)^{2}\right.\right. \\
& \left.\left(1+\left(\frac{914}{\left(D_{e}-2 \varepsilon\right)}+730494\left(\frac{\mu_{\text {gas }}\left(D_{e}-2 \varepsilon\right) N}{\dot{m}_{\text {gas }}}\right)^{1,3}\right)^{0,5}\right]\right) \\
& -\dot{m}_{\text {air }} R_{\text {air }} \ln \left[1-\frac{1,93}{\rho_{\text {air }} P_{\text {air }, e}}\left(\frac{\mu_{\text {air }}}{D_{e}}\right)^{0,25}\left(\frac{3 N}{N_{A}}+1\right)\right. \\
& \left.\left.\left(\frac{1-D_{e} / \sqrt{\left.S_{T} / 2\right)^{2}+S_{L}^{2}}}{S_{T} / D_{e}-1}\right)^{0,5}\left(\frac{\dot{m}_{\text {air }}}{\left(S_{T}-D_{e}\right)(L / 3) N_{A}}\right)^{1,75}\right]\right\}
\end{aligned}
$$


La función objetivo se considera acotada por las siguientes restricciones:
a) $0.01<D_{e}<0.15$
b) $S_{T}>D_{e}$
c) $S_{T}^{T} / S_{L}<2$
d) $N_{A}\left(D_{e}+s_{T}\right)<B_{\text {cal }}$
e) $D_{e}<\sqrt{s_{T}^{2}+s_{L}^{2}}$
f) $1-\frac{D e}{s_{L}}$$$
\frac{s_{L}}{\frac{s_{T}}{D_{e}}-1}>0,53
$$

\section{Caso estudio (Resultados y análisis de resultados)}

La función objetivo es continua dentro del rango de análisis, tiene un fuerte comportamiento no lineal, por lo tanto, para resolverla se utilizó el Toolbox de Optimización del Software MATLAB 6.5, a través de la función FMINCON, que utiliza un algoritmo de media escala, la Programación Secuencial Cuadrática (POS) y el método Cuasi-Newton.

Se introducen al problema los datos mostrados en la tabla 1. Los costos de irreversibilidad asociados a diferencia de temperatura y caída de presión se obtienen de Marín (2003).

Los valores óptimos de las variables libres de diseño se comparan con el calentador de aire instalado en la caldera RETO 45-28 en la tabla 2. Algunos de los parámetros óptimos importantes se listan a continuación y se comparan con el calentador de aire instalado en la caldera RETO 45-28 en la tabla 3.

Dentro de los resultados se observa que, para las consideraciones realizadas, es recomendable ocupar todo el ancho de la caldera con tubos, así mismo, utilizar la máxima longitud posible de los tubos, hasta donde el espacio del generador de vapor lo permita; el diámetro de los tubos del CA optimizado y el diseño tradicional son iguales para los pasos transversal y longitudinal dados; el número de tubos se incrementa considerablemente para el diseño optimizado, así también, aumenta el número de tubos en la profundidad del equipo de 28 a 50 unidades; las velocidades del aire y gases disminuyen como una consecuencia del incremento de las áreas transversales de paso del aire y gases, de tal forma que el coeficiente global de transferencia de calor disminuye de 29,18 a 20,30 W/(m2 K); los NTU del diseño optimizado pasan de 2,51 a 7,22 y el área total de transferencia de calor se cuadruplica. Se observa que para el primer caso, la irreversibilidad provocada por la caída de presión es sólo 3\% de la total generada, mientras que en el diseño optimizado pasa a ser $1,9 \%$, todo esto provoca un decremento en los costos de operación del equipo de 1,97 US\$/s al compararse con el de la caldera RETO 45-28.

Tabla 1. Datos del calentador de aire (continúa...)

\begin{tabular}{|c|c|c|c|}
\hline Nombre & Símbolo & Cantidad & Unidades \\
\hline Espesor de tubos & $\varepsilon$ & $1,6 \times 10^{-3}$ & $\mathrm{~m}$ \\
\hline Paso transversal & $S_{T}$ & 0,06 & $\mathrm{~m}$ \\
\hline Paso longitudinal & $S_{L}$ & 0,04 & $\mathrm{~m}$ \\
\hline Conductividad del acero & $k_{A C}$ & 50,2 & $\mathrm{~W} /\left(\mathrm{m}^{\circ} \mathrm{C}\right)$ \\
\hline Conductividad de gases & $k_{\text {gas }}$ & 0,0401 & $\mathrm{~W} /\left(\mathrm{m}^{\circ} \mathrm{C}\right)$ \\
\hline Conductividad de aire & $k_{\text {air }}$ & 0,0393 & $\mathrm{~W} /\left(\mathrm{m}^{\circ} \mathrm{C}\right)$ \\
\hline Calor específico de gases & $c_{p, g a s}$ & 1,19 & $\mathrm{~kJ} /(\mathrm{kg} \mathrm{K})$ \\
\hline Calor específico de aire & $c_{p, a i r}$ & 1,005 & $\mathrm{~kJ} /(\mathrm{kg} \mathrm{K})$ \\
\hline Viscosidad dinámica de gases & $\mu_{\text {gas }}$ & $24,5 \times 10^{-6}$ & Pa.s \\
\hline Viscosidad dinámica de aire & $\mu_{\text {air }}$ & $26 \times 10^{-6}$ & Pa.s \\
\hline Flujo de gases & $\dot{m}_{\text {gas }}$ & 29,5 & $\mathrm{~kg} / \mathrm{s}$ \\
\hline
\end{tabular}


DOI: http://dx.doi.org/10.22201/fi.25940732e.2009.10n4.026

J.J. Marín-Hernández, M.L. González-PetitJean y O.M. Cruz-Fonticiella

Tabla 1. Datos del calentador de aire (...continuación)

\begin{tabular}{lccc}
\hline \multicolumn{1}{c}{ Nombre } & Símbolo & Cantidad & Unidades \\
\hline Flujo de aire & $\dot{m}_{\text {air }}$ & 17,48 & $\mathrm{~kg} / \mathrm{s}$ \\
Costo de irr. por diferencia de temp. del CA & $c_{l}^{\Delta T}$ & $3.36 \times 10^{-6}$ & $\$ / \mathrm{kJ}$ \\
Costo de irr. por caída de presión del CA & $c_{l}^{\Delta P}$ & $16 \times 10^{-6}$ & $\$ / \mathrm{kJ}$ \\
Temperatura de referencia & $T_{0}$ & 298,15 & $\mathrm{~K}$ \\
Temperatura de gases de entrada & $T_{\text {gas }, \text { en }}$ & 609,75 & $\mathrm{~K}$ \\
Temperatura de aire de entrada & $T_{\text {air,en }}$ & 298,15 & $\mathrm{~K}$ \\
Rugosidad del tubo & Rug & $45,7 \times 10^{-6}$ & $\mathrm{~m}$ \\
Constante R de gases & $R_{\text {gas }}$ & 0,3023 & $\mathrm{~kJ} /(\mathrm{kg} \mathrm{K})$ \\
Constante R de aire & $R_{\text {air }}$ & 0,287 & $\mathrm{~kJ} /(\mathrm{kg} \mathrm{K})$ \\
Densidad de gases & $\rho_{\text {gas }}$ & 0,748 & $\mathrm{~kg} / \mathrm{m}^{3}$ \\
Densidad de aire & $\rho_{\text {air }}$ & 0,746 & $\mathrm{~kg} / \mathrm{m}^{3}$ \\
Presión de salida de gases & $P_{\text {gas,s }}$ & 98806 & $\mathrm{~Pa}$ \\
Presión de entrada de aire & $P_{\text {air,e }}$ & 102809 & $\mathrm{~Pa}$ \\
Ancho de la caldera & $B_{\text {CAL }}$ & 6,84 & $\mathrm{~m}$ \\
\hline
\end{tabular}

Tabla 2. Parámetros geométricos óptimos

\begin{tabular}{cccc}
\hline Variable & $\begin{array}{c}\text { Sistema } \\
\text { RETO 45-28 }\end{array}$ & Sistema óptimo & Unidades \\
\hline$D_{e}$ & 0.04 & 0.04 & $\mathrm{~m}$ \\
$L$ & 3,6 & 10 & $\mathrm{~m}$ \\
$N$ & 3192 & 5000 & tubos \\
$N_{A}$ & 114 & 100 & tubos \\
\hline
\end{tabular}

Tabla 3. Comparación de parámetros del calentador de aire entre el diseño tradicional y el diseño optimizado termoeconómicamente (continúa...)

\begin{tabular}{|c|c|c|c|}
\hline Variable & $\begin{array}{c}\text { Sitema } \\
\text { RETO 45-28 }\end{array}$ & Sistema óptimo & Unidades \\
\hline Número de tubos en la profundidad & 28 & 50 & tubos \\
\hline Área transversal de gases & 3,39 & 6,22 & $\mathrm{~m}^{2}$ \\
\hline
\end{tabular}


Tabla 3. Comparación de parámetros del calentador de aire entre el diseño tradicional y el diseño optimizado termoeconómicamente (...continuación)

\begin{tabular}{|c|c|c|c|}
\hline Variable & $\begin{array}{c}\text { Sitema } \\
\text { RETO 45-28 }\end{array}$ & Sistema óptimo & Unidades \\
\hline Velocidad de gases & 14,35 & 7,50 & $\mathrm{~m} / \mathrm{s}$ \\
\hline Número de Reynolds de flujo gases & 13051 & 8380 & - \\
\hline Coeficiente de convección lado gases & 51,78 & 31,25 & $\mathrm{~W} /\left(\mathrm{m}^{2 \circ} \mathrm{C}\right)$ \\
\hline Área transversal de aire & 3,17 & 6,74 & $\mathrm{~m}^{2}$ \\
\hline Velocidad de aire & 6,35 & 3,48 & $\mathrm{~m} / \mathrm{s}$ \\
\hline Número de Reynolds de flujo de aire & 9829 & 3970 & - \\
\hline Coeficiente de convección lado aire & 61,63 & 14,12 & $\mathrm{~W} /\left(\mathrm{m}^{2 \circ} \mathrm{C}\right)$ \\
\hline Coeficiente global de transferencia de calor & 29,18 & 20,30 & $\mathrm{~W} /\left(\mathrm{m}^{2 \circ} \mathrm{C}\right)$ \\
\hline Área de transferencia de calor & 1385 & 6250 & $\mathrm{~m}^{2}$ \\
\hline NTU & 2,51 & 7,22 & - \\
\hline Irreversibilidad por diferencia de temperatura & 762,58 & 652,5 & $\mathrm{~kW}$ \\
\hline Irreversabilidad por caída de presión & 23,63 & 12,58 & $\mathrm{~kW}$ \\
\hline Costo de irr. por diferencia de temperatura & 9,22 & 7,89 & $\$ / s$ \\
\hline Costo de irrr. por caída de presión & 1,36 & 0,72 & $\$ / s$ \\
\hline Costo de operación & 10,59 & 8,62 & $\$ / s$ \\
\hline
\end{tabular}

\section{Nomenclatura}

A Área de transferencia de calor [m2]

$A_{\text {gas }}$ Área transversal del flujo de gases [m2]

$A_{\text {air }}^{\text {gas }}$ Área transversal del flujo de aire [m2]

Arr Arreglo

$B_{C A L}$ Ancho de caldera [m]

C Costo total de operación del sistema global [\$], Capacidad calorífica $[\mathrm{kJ} /(\mathrm{s}$

$\mathrm{K})$ ], razón de capacidades caloríficas [adimensional]

$c_{p} \quad$ Calor específico a presión constante $[\mathrm{kJ} /(\mathrm{kg} \mathrm{K})]$

c) Costo por irreversibilidad [\$/kJ]

$D_{e} \quad$ Diámetro exterior de tubos [m]

$D_{i} \quad$ Diámetro interior de tubos [m]

Eu Número de Euler [adimensional]

he Coeficiente de transferencia de calor por convección, lado externo $\left[\mathrm{W} / \mathrm{m} 2{ }^{\circ} \mathrm{C}\right]$

hi Coeficiente de transferencia de calor por convección, lado interno $\left[\mathrm{W} / \mathrm{m} 2{ }^{\circ} \mathrm{C}\right]$

I Irreversibilidad $[\mathrm{kJ}]$
$I_{T} \quad$ Irreversibilidad total $[\mathrm{kJ}]$

$k \quad$ Conductividad de térmica $\left[\mathrm{W} /\left(\mathrm{m}^{\circ} \mathrm{C}\right)\right]$

$L \quad$ Longitud de tubos [m]

Min Minimizar

$m$ Masa $[\mathrm{kg}]$

$N \quad$ Número total de tubos [tubos]

$N_{A}$ Número de tubos a lo ancho de la caldera [tubos]

$N_{p} \quad$ Número de tubos a lo profundo [tubos]

$\mathrm{Nu}$ Número de Nusselt [adimensional]

NTUNúmero de unidades de transferencia [adimensional]

$P \quad$ Presión $[\mathrm{MPa}]$

Pr Número de Prandtl [adimensional]

$R \quad$ Constante particular del gas [kJ/(kg K)]

$\operatorname{Re}$ Número de Reynolds [adimensional]

Rug Rugosidad de tubos [m]

$S_{T} \quad$ Paso transversal [m]

$S_{L} \quad$ Paso longitudinal [m]

$T$ Tempertura $\left[{ }^{\circ} \mathrm{C}\right]$

$U$ Coeficiente global de transferencia de calor $\left[\mathrm{W} /\left(\mathrm{m} 2{ }^{\circ} \mathrm{C}\right)\right]$ 
$V \quad$ Velocidad $[\mathrm{m} / \mathrm{s}]$

$Z$ Relación de número de tubos [adimensional]

\section{Griegos}

$\Delta \quad$ Gradiente

$\varepsilon \quad$ Espesor de tubo [m]

$\in$ Eficacia [\%]

$\lambda \quad$ Factor de fricción [adimensional]

$\mu \quad$ Viscosidad dinámica [Pa.s]

$v \quad$ Viscosidad cinemática $[\mathrm{m} 2 / \mathrm{s}]$

$\rho \quad$ Densidad $[\mathrm{kg} / \mathrm{m} 3]$

AC Acero

$0 \quad$ Estado del medio ambiente

air Aire

e Corriente de entrada

gas Gases de combustión

s Corriente de salida

\section{Superíndices}

$\Delta T$ Asociado a la diferencia de temperatura

$\Delta P \quad$ Asociado a la caída de presión

- Variable por unidad de tiempo

\section{Anexos}

Ecuaciones utilizadas para determinar la IDT en función de los parámetros geométricos de un calentador de aire tubular vertical de flujo cruzado a contracorriente de tres pasos instalado en una caldera bagacera.

$$
\begin{aligned}
& C_{\text {air }}<C_{\text {gas }} \\
& C_{\text {air }}=C_{\min } \\
& \in=\frac{T_{\text {air }, s}-T_{\text {air }, e}}{T_{\text {gas }, e}-T_{\text {air }, e}} \\
& C_{g a s}\left(T_{\text {gas }, e}-T_{\text {gas }, s}\right)=C_{\text {air }}\left(T_{\text {air }, s}-T_{\text {air }, e}\right) \\
& C=\frac{C_{\min }}{C_{\max }} \\
& \in=\frac{1-\operatorname{Exp}[-N T U(1-C)]}{1-C \operatorname{Exp}[-N T U(1-C)]} \\
& N T U=\frac{U A}{C_{\min }} \\
& A=\pi D_{e} L N
\end{aligned}
$$

$$
U=\left[\frac{1}{h_{i}}+\frac{D_{i} \ln \left(D_{e} / D_{i}\right)}{2 k_{A C}}+\frac{D_{e}}{D_{i} h_{e}}\right]^{-1}
$$

\section{Análisis lado gases}

$$
\begin{aligned}
& N u_{\text {gas }}=0.023 \operatorname{Re}_{\text {gas }}^{0,8} \operatorname{Pr}_{\text {gas }}^{0,3} \\
& \operatorname{Re}_{\text {gas }}=\frac{V_{\text {gas }} D_{i}}{v_{\text {gas }}} \\
& v_{\text {gas }}=\frac{\mu_{\text {gas }}}{\rho_{\text {gas }}} \\
& \operatorname{Pr}_{\text {gas }}=\frac{c_{p, g a s} \mu_{\text {gas }}}{k_{\text {gas }}} \\
& \dot{m}_{g a s}=\rho_{g a s} V_{g a s} A_{g a s} \\
& A_{\text {gas }}=\frac{\pi D_{i}^{2} N}{4}
\end{aligned}
$$

$$
\begin{aligned}
& N=N_{A} N_{P} \\
& N u_{\text {gas }}=\frac{h_{i} D_{i}}{k_{\text {gas }}}
\end{aligned}
$$

Análisis lado aire

$$
N u_{\text {air }}=0.41 \operatorname{Re}_{\text {air }}^{0,6} \operatorname{Pr}_{\text {air }}^{0,33}\left(\frac{s_{T}}{s_{L}}\right)^{1 / 6} s i \frac{S_{T}}{S_{L}}<2
$$

$$
N u_{\text {air }}=\frac{h_{e} D_{e}}{k_{\text {air }}}
$$




$$
A_{\text {air }}=\left(s_{T}-D_{e}\right)\left(\frac{L}{3}\right) N_{A}
$$

Ecuaciones utilizadas para determinar la ICP en función de los parámetros geométricos de un calentador de aire tubular de flujo cruzado a contracorriente de tres pasos instalado en una caldera bagacera.

\section{Análisis lado gases}

$$
\begin{aligned}
& \Delta P_{g a s}=\lambda \rho_{g a s} \frac{L}{\left(D_{e}-\varepsilon\right)} \frac{V_{g a s}^{2}}{2} \\
& \Delta P_{g a s}=P_{g a s}-P_{g a s, s} \\
& \lambda=0,0055\left[1+\left(20000 \frac{R u g}{\left(D_{e}-\varepsilon\right)}+\frac{10^{6}}{\operatorname{Re}^{1,3}}\right)^{0,5}\right]
\end{aligned}
$$

Análisis lado aire

$$
\begin{aligned}
& \Delta P_{\text {air }}=P_{\text {air }, e}-P_{\text {air }, s} \\
& \Delta P_{\text {air }}=E u \rho_{\text {air }} V_{\text {air }}^{2} \\
& E u=1,93\left(3 \frac{N}{N_{A}}+1\right)\left(\frac{1-\frac{D_{e}}{\sqrt{s_{T}^{2}-s_{L}^{2}}}}{\frac{s_{T}}{D_{e}}-1}\right)^{0,5} \operatorname{Re}^{-0,25}
\end{aligned}
$$

para

$$
\frac{1-\frac{D_{e}}{S_{L}}}{\frac{S_{T}}{D_{e}}-1}>0,53
$$

profundidad para conformar un total de 5000 tubos. De esta forma, el coeficiente global de transferencia de calor se reduce a $20,30 \mathrm{~W} /(\mathrm{m} 2 \mathrm{~K})$, los NTU se incremen$\tan$ a 7,22 y las velocidades de gases y aire se reducen a 7,50 y $3,48 \mathrm{~m} / \mathrm{s}$ respectivamente. Así, el diseño del CA optimizado geométricamente provoca un decremento en los costos de operación del equipo de 1,97 US\$/s al compararse con el de la caldera RETO 45-28.

\section{Referencias}

Cornelissen R.L. Thermodynamics and Sustainable Development. (PhD. Thesis). The Netherlands. Twente University. 1997.

Gong M., Wall G. On Exergetics Economics and Optimization of Technical Processes to Meet

Environmental Conditions. Presented at TAIES '97, Beijing, China, June 10-13, pp. 453-460. 1997.

Holman P.J. Transferencia de calor. Octava Edición. España. Mc Graw-Hill. 1998.

Hugot E. Handbook on Cane Sugar Engineering. Third edition. New York. Elsevier Science Publishing Company Inc. 1986.

Incropera F.P., De Witt D.P. Fundamentals of Heat and Mass Transfer. $4^{\text {th }}$ edition. USA. Wiley and Sons. 1996.

Marín J.J. Optimización termoeconómica para el diseño de recuperadores de calor instalados en calderas bagaceras. Tesis (Doctorado). Santa Clara, Cuba. Centro de Estudios Energéticos y Tecnologías Ambientales, Facultad de Mecánica, Universidad Central de las Villas. 2003.

Valero A. Termoeconomía: El punto de encuentro de la termodinámica, la economía y la ecología. CIRCE, Departamento de Ingeniería Mecánica. Universidad de Zaragoza. Noviembre 1997.

\section{Conclusiones}

La optimización bajo una perspectiva termoeconómica de las variables geométricas de los CA del tipo tubular vertical con flujo cruzado a contracorriente, los cuales son típicos de calderas bagaceras, propone novedosamente algunos cambios en la construcción tradicional. Para las consideraciones realizadas se recomienda: mantener el diámetro en $40 \mathrm{~mm}$; hasta donde los espacios de la caldera lo permitan, utilizar tubos más largos (10 m); colocar 100 tubos a lo ancho de la caldera y 50 en la 